1997-9

\title{
Local Refinement of Three-Dimensional Finite Element Meshes
}

\author{
M. L. Staten \\ ANSYS, Inc. \\ Norman L. Jones \\ Brigham Young University - Provo, njones@byu.edu
}

Follow this and additional works at: https://scholarsarchive.byu.edu/facpub

Part of the Geotechnical Engineering Commons

\section{Original Publication Citation}

Staten, Matthew L., and Norman L. Jones, "Local Refinement of Three-Dimensional Finite Element Meshes,"Engineering With Computers, 1997,Vol. 13, pp. 165-174.

\section{BYU ScholarsArchive Citation}

Staten, M. L. and Jones, Norman L., "Local Refinement of Three-Dimensional Finite Element Meshes" (1997). Faculty Publications. 4300.

https://scholarsarchive.byu.edu/facpub/4300

This Peer-Reviewed Article is brought to you for free and open access by BYU ScholarsArchive. It has been accepted for inclusion in Faculty Publications by an authorized administrator of BYU ScholarsArchive. For more information, please contact ellen_amatangelo@byu.edu. 


\title{
Local Refinement of Three-Dimensional Finite Element Meshes
}

\author{
M.L. Staten ${ }^{1}$ and N.L. Jones ${ }^{2}$ \\ ${ }^{1}$ ANSYS, Inc., Houston, Houston, PA, USA; ${ }^{2}$ Brigham Young University, Provo, Utah, USA
}

\begin{abstract}
Mesh refinement is an important tool for editing finite element meshes in order to increase the accuracy of the solution. Refinement is performed in an iterative procedure in which a solution is found, error estimates are calculated, and elements in regions of high error are refined. This process is repeated until the desired accuracy is obtained.

$M u c h$ research has been done on mesh refinement. Research has been focused on two-dimensional meshes and threedimensional tetrahedral meshes ([1] Ning et al. (1993) Finite Elements in Analysis and Design, 13, 299-318; [2] Rivara, M. (1991) Journal of Computational and Applied Mathematics 36, 79-89; [3] Kallinderis; Vijayar (1993) AIAA Journal, 31, 8, 1440-1447; [4] Finite Element Meshes in Analysis and Design, 20, 47-70). Some research has been done on three-dimensional hexahedral meshes ([5] Schneiders; Debye (1995) Proceedings IMA Workshop on Modelling, Mesh Generation and Adaptive Numerical Methods for Partial Differential Equations). However, little if any research has been conducted on a refinement algorithm that is general enough to be used with a mesh composed of any threedimensional element (hexahedra, wedges, pyramids, and/or tetrahedra) or any combination of three-dimensional elements (for example, a mesh composed of part hexahedra and part wedges). This paper presents an algorithm for refinement of three-dimensional finite element meshes that is general enough to refine a mesh composed of any combination of the standard three-dimensional element types.
\end{abstract}

Keywords. Mesh refinement; Mixed element meshes; Unstructured meshes

\section{Introduction}

Mesh refinement is the process of taking an existing finite element mesh and changing the size, shape, and/or order of the elements that compose the mesh to increase the accuracy of the solution. Elements that are good candidates for refinement are elements in regions where high error is found or large gradients

Correspondence and offprint requests to: Dr N.L. Jones, $368 \mathrm{CB}$, Brigham Young University, Provo, Utah 84602, USA. are expected. Some solvers are equipped with automatic refinement capabilities. An iterative procedure is performed in which a solution is found using an existing mesh, error estimates are calculated, and elements in regions of high error are refined. This process is repeated until the desired accuracy is obtained. Many solvers, however, are not equipped with automated refinement capabilities. In such cases, it becomes necessary to manually select a set of elements and refine the selected elements external to the solver. The algorithms presented in this paper are general enough to be implemented either external to or within any iterative finite element solver.

Three methods of refinement are presented. The first is called all-elements-to-tetrahedra and converts the elements in the regions of refinement into tetrahedra, regardless of the original element types. The second method is called retain-element-types and attempts to convert the elements in the regions of refinement into smaller elements of the same type as the original elements. The third method is called all-elements-totetrahedra-coarse and is a variation of the allelements-to-tetrahedra method of refinement.

\section{Previous Research}

The algorithms presented in this paper are extensions of the algorithm presented in Schneiders and Debye [5]. Schneiders and Debye developed a twodimensional refinement algorithm and extended it to three-dimensional hexahedral elements. Figure 1 illustrates the two-dimensional mesh refinement algorithm presented by Schneiders and Debye. In Fig. 1(a), elements $A, B$ and $C$ have been selected for refinement. The two-dimensional algorithm starts by replacing each of the elements selected for refinement by one of the refinement templates in Fig. 2 resulting in the mesh shown in Fig. 1(b). 




Fig. 1. Two-dimensional mesh refinement.

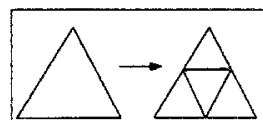

(a)

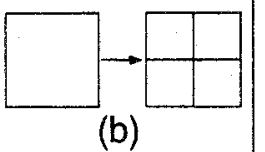

(b)

Fig. 2. Selected element refinement templates.

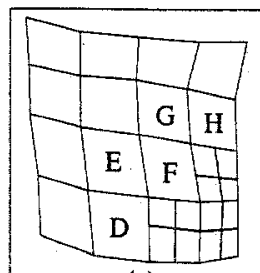

(a)

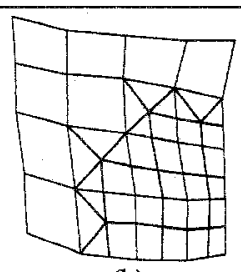

(b)
Fig. 3. Refinement of transition elements.

The algorithm then proceeds by identifying transition elements. A transition element is defined as any element that shares one or more nodes with elements that were selected for refinement. Transition elements must be identified and refined in order to eliminate the disjoint edges between elements that were selected for refinement and elements not selected for refinement (Fig. 1(b)). Elements D, E, F, G and H in Fig. 3(a) each share at least one node with an element selected for refinement and are therefore identified as transition elements. Each transition element is refined by replacing it with one of the transition element templates found in Fig. 4. To decide which of the eight transition templates should be used, it is necessary to determine which of the nodes that define the transition element are shared with an element that was selected for refinement. For example, element $\mathrm{E}$ in Fig. 3 shares one node with an element that was selected for refinement (element $A$ in Fig. 1(a)) and is therefore replaced with the transition template defined in Fig. 4(a). Likewise, element F in Fig. 3 shares three nodes with elements that were selected for refinement and is therefore replaced with the transition template defined in Fig. 4(d) $[5,6]$.



(a)

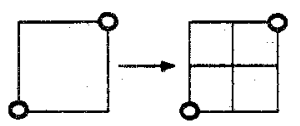

(c)

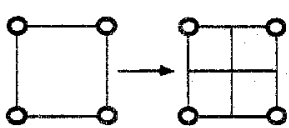

(e)

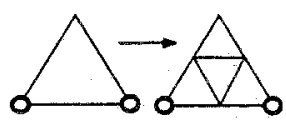

(g)

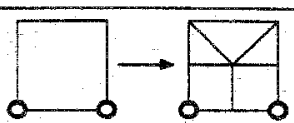

(b)

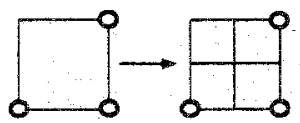

(d)

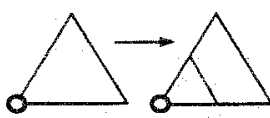

(f)

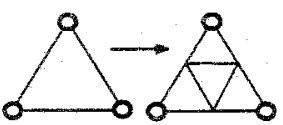

(h)
Fig. 4. Transition element templates for two-dimensional refinement.

The algorithm presented in Figs 1 and 3 is what Schneiders and Debye refer to as two-refinement. Schneiders and Debye also present a similar algorithm called three-refinement. Three-refinement has the advantage that no triangles are introduced when refining quad meshes. Two-refinement, however, is much easier to extend into three-dimensional refinement. The three-dimensional algorithm presented in the next section is simply an extension of two-refinement from two-dimensions to threedimensions.

\section{Three-Dimensional Mesh Refinement}

The three-dimensional refinement algorithm presented here is an extension of the two-dimensional tworefinement algorithm presented above and by Schneiders and Debye. Schneiders and Debye extended the two-refinement algorithm into three dimensions for hexahedral elements. The algorithm presented in this paper extends two-refinement into three dimensions for hexahedra, wedges, pyramids and tetrahedra.

Figure 5 summarizes the algorithm used for three-dimensional refinement. The only difference between the two-dimensional algorithm and the three-dimensional algorithm is the templates used for selected and transition elements. In two dimensions, there are a total of eight transition element templates that must be used. In three dimensions, however, there are 52 transition element templates. 


Steps in Three-Dimensional Refinement
Algorithm
1. Select the elements that are to be refined.
2. Refine the elements selected for refinement
appropriate refinement template.
3. Identify the transition elements (i.e. Any
element that shares a node with an element
that was refined in step 2 ).
Loop through the transition elements
identified in step 3. For each element,
determine which transition element template
trom Figure 7 applies. Replace the
transition element with the appropriate

Fig. 5. Summary of steps in 3D refinement.

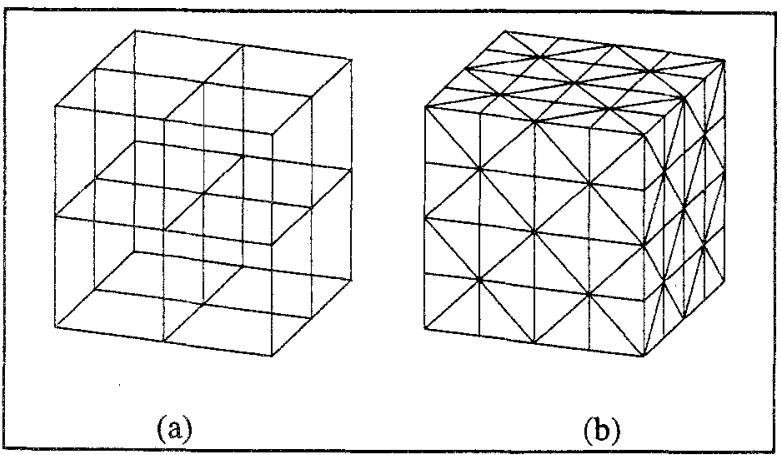

Fig. 6. An all hex mesh refined using the all-elements-to-tetrahedra method.

\section{All-Elements-to-Tetrahedra Refinement}

The simplest form of three-dimensional mesh refinement is to subdivide all of the elements that are selected for refinement into tetrahedra. Consider the simple hexahedral mesh shown in Fig. 6(a). To refine this mesh using the all-elements-to-tetrahedra method, each of the hexahedra is subdivided into tetrahedra. Figure 6(b) shows the result of refining the mesh in Fig. 6(a) using the all-elements-to-tetrahedra method.

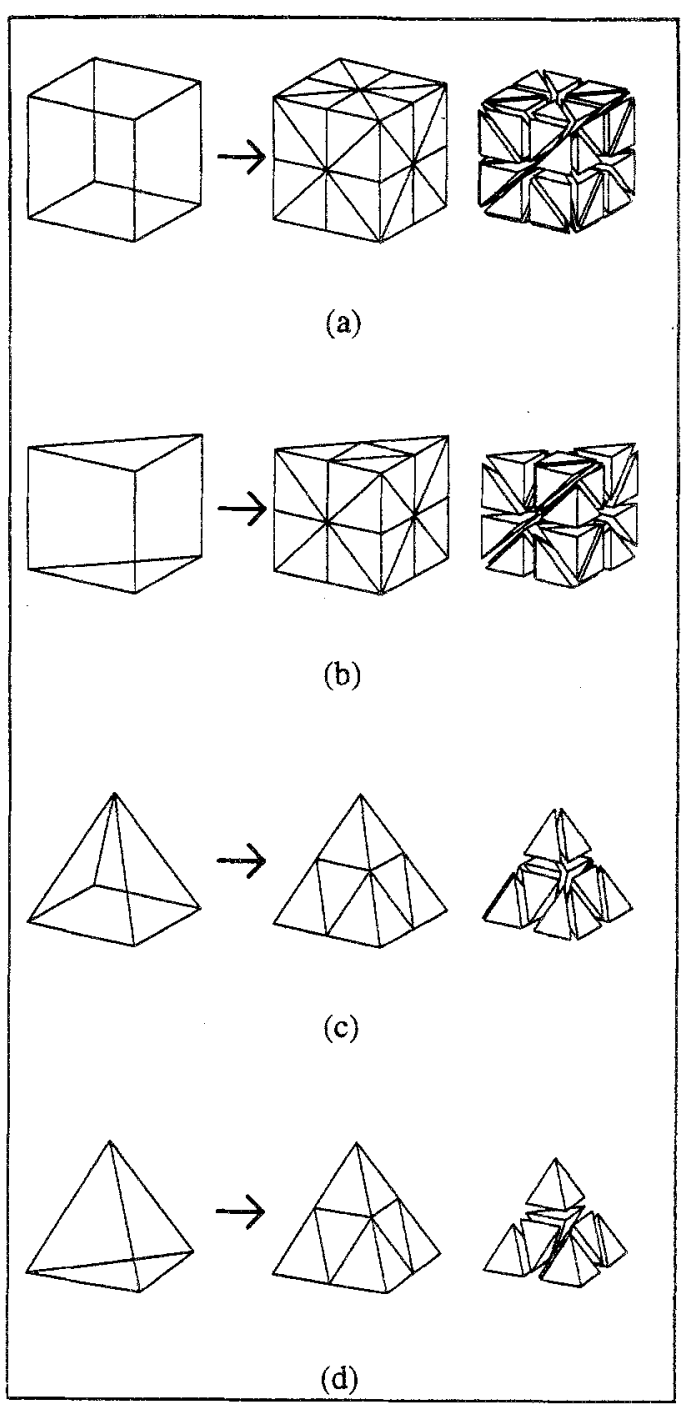

Fig. 7. 3D selected element refinement templates for the allelements-to-tetrahedra method.

Each hexahedron was subdivided into tetrahedra by replacing it with the refinement template in Fig. 7(a). Figures 7(b), 7(c) and 7(d) show the refinement templates used if the mesh is composed of any of the other standard element types. Each template changes the original element into tetrahedra. No transition element templates were used in the refinement shown in Fig. 6, because all of the elements in the mesh were refined.

Figure 8 shows the same mesh as in Fig. 6(a) with only one of the original elements (element A) being selected for refinement. Figure 9 shows the transition element templates used to refine the mesh in Fig. 8(a). Three of the transition elements share one complete face with element $\mathrm{A}$ in Fig. 8(a) and are replaced with the transition element template found in Fig. 9(c). Three of the transition elements share one 


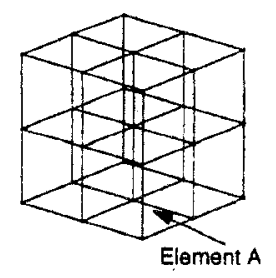

(a)



(b)
Fig. 8. All hex mesh partially refined using the all-elements-totetrahedra method.

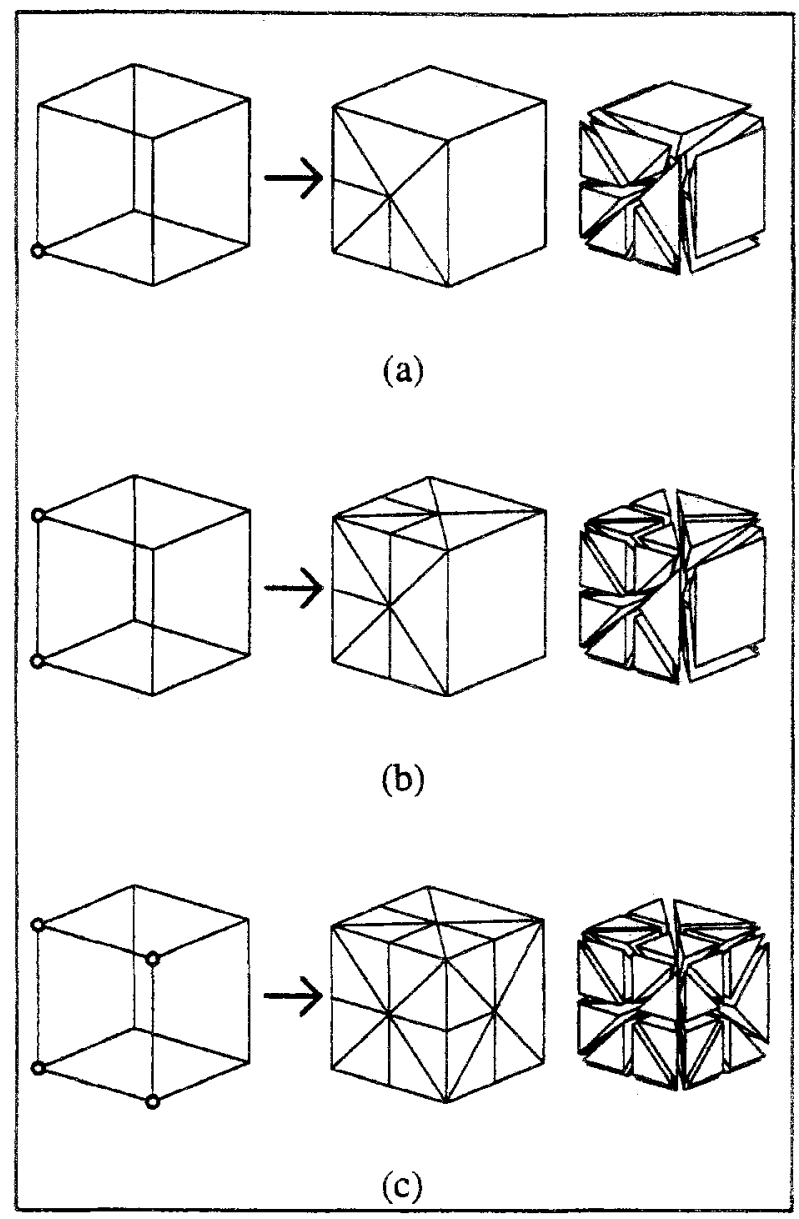

Fig. 9. 3D hexahedral transition element templates for the all-elements-to-tetrahedra method.

complete edge with element $\mathrm{A}$ in Fig. 8(a) and are replaced with the transition element template found in Fig. 9(b). One of the transition elements shares a single node with element A in Fig. 8(a) and is replaced with the transition element template found in Fig. 9(a).

Although the case does not arise in the example shown in Fig. 8, it could be the case that a transition element is completely surrounded by elernents which were selected for refinement. If this is the case, all of the nodes that define the transition element are shared with elements that were selected for refinement and the transition element is refined as if it were also selected for refinement.

The sub-elements of all selected element refinement templates (Fig. 7) are composed of entirely tetrahedra, The same is not true, however, for the transition element templates (Fig. 9). It is impossible to maintain strictly tetrahedra in the transition regions of meshes originally composed of elements other than tetrahedra. Figure 9 illustrates three of the 52 required transition element templates for the all-elements-to-tetrahedra method of refinement. The transition element templates not included in this paper are used to refine the transition elements in tetrahedral, pyramid, wedge and other combinations of hexahedral elements. It is impractical to include all 52 templates in this paper. Each of the 52 templates for the all-elements-totetrahedra method, however, has been defined and illustrated in detail in Staten [6]. Staten includes a complete listing of the topology of each of the sub-elements in the transition elements.

\section{Retain-Element-Types Refinement}

Another form of three-dimensional mesh refinement is to subdivide all of the elements that are selected for refinement into smaller elements of the same type. Consider the simple hexahedral mesh shown in Fig. 10(a). To refine this mesh using the retain-elementstypes method, each of the hexahedra is subdivided into eight smaller hexahedra. Figure 10(b) shows the result of refining the mesh in Fig. 10(a) using the retain-element-types method. Each hexahedra was subdivided into smaller hexahedra by replacing it with the refinement template in Fig. 11(a). Figures 11(b-d) show the refinement templates used if the mesh is composed of any of the other standard element types. Each template, with the exception of the pyramid

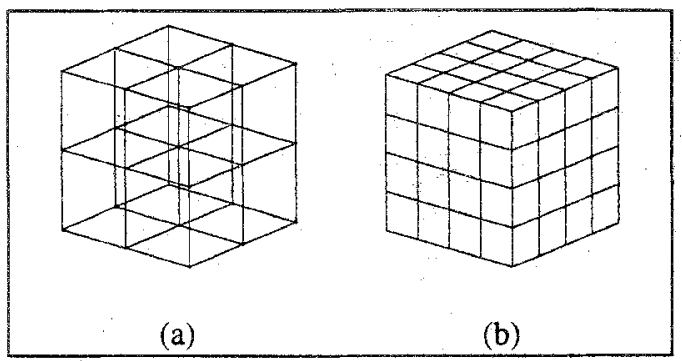

Fig. 10. A hexahedral mesh refined using the retain-element-types method of refinement. 


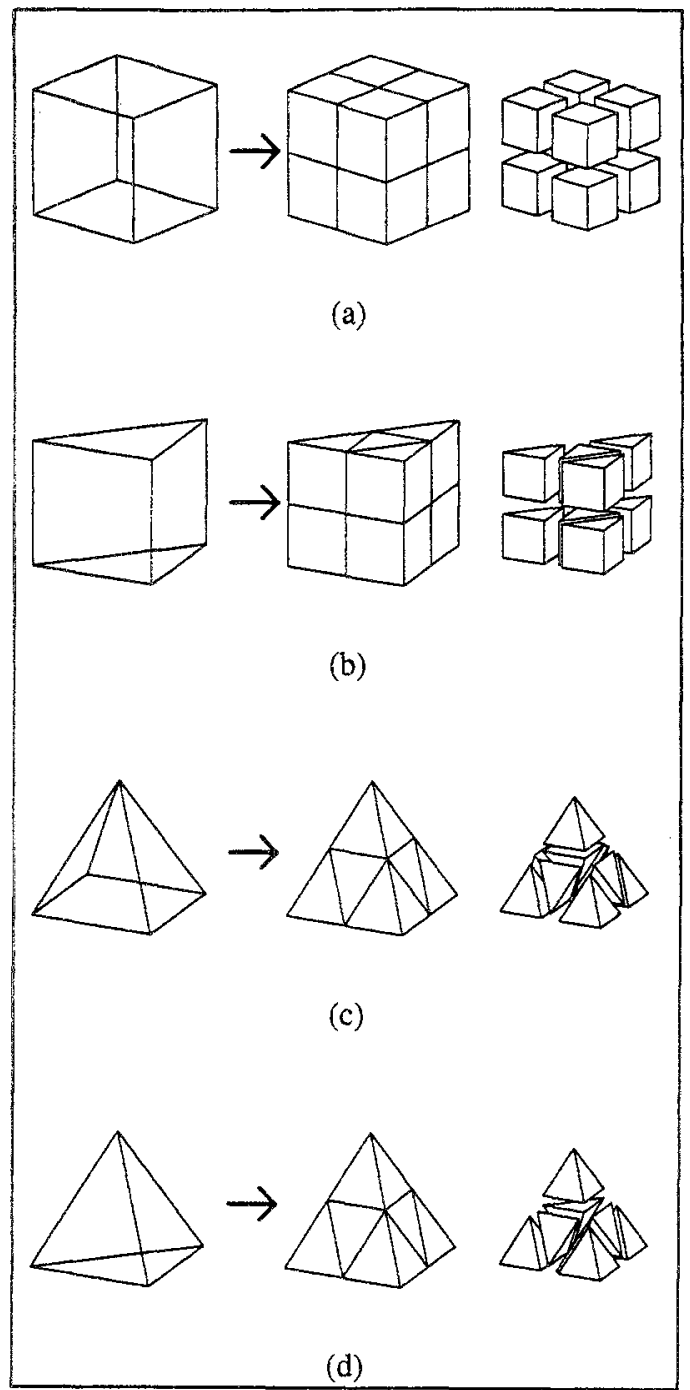

Fig. 11. 3D selected element refinement templates for retainelements-types.

template replaces the original element with smaller elements of the same type. No transition element templates were used in the refinement shown in Fig. 10 because all of the elements in the mesh were refined.

Figure 12 shows the same mesh as in Fig. 6(a) with only one of the original elements (element A) being selected for refinement. Figure 13 shows the transition element templates used to refine the mesh in Fig. 12(a). Three of the transition elements share one complete face with element A in Fig. 12(a) and are replaced with the transition element template found in Fig. 13(c). Three of the transition elements share one complete edge with element A in Fig. 12(a) and are replaced with the transition element template found in Fig. 13(b). One of the transition elements shares a single node with element A in Fig. 12(a) and is replaced

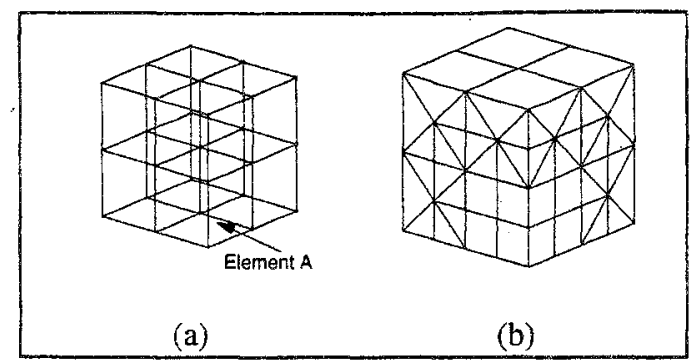

Fig. 12. An all hex mesh refined using the retain-element-types method.

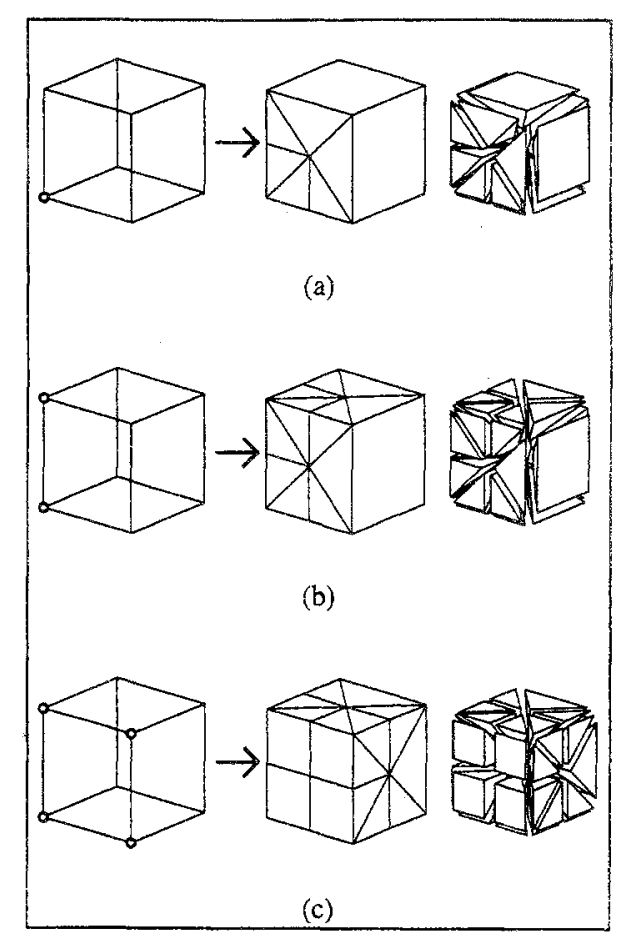

Fig. 13. 3D hexahedral transition element templates for the retain-element-types method.

with the transition element template found in Fig. 13(a).

Figure 13 illustrates three of the 52 required transition element templates for the retain-elementstypes method of refinement. The transition element templates not included in this paper are used to refine the transition elements in tetrahedral, pyramid, wedge and other combinations of hexahedral elements. As with the templates for the all-elements-to-tetrahedra method of refinement, it is impractical to include all 52 templates in this paper. Each of the 52 templates for the retain-element-types method, however, has been defined and illustrated in detail in Staten [6]. Staten includes a complete listing of the topology of each of the sub-elements in the transition elements. 


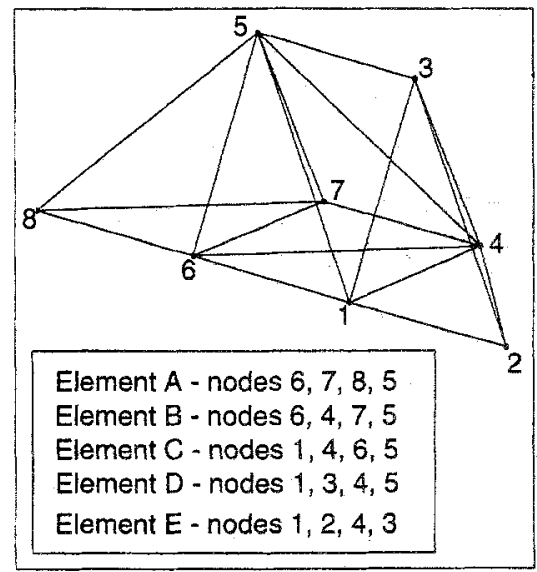

Fig. 14. A five element tetrahedral mesh.

All of the selected element refinement templates, with the exception of the pyramid refinement template (Fig. 11), refine the selected elements in such a way that the original elements are replaced with smaller elements of the same type. However, with the exception of tetrahedral meshes, the original element types are not completely maintained in the transition region.

To illustrate maintaining tetrahedra in the transition region of a mesh composed entirely of tetrahedra, consider the tetrahedral mesh found in Fig. 14. Element $A$ is selected and refined by replacing it with the template defined in Fig. 11(d). Element B shares three nodes with element $A$ and is therefore replaced with the template found in Fig. 15(c). Element $C$ shares two nodes with element $A$ and is therefore replaced with the template found in Fig. 15(b). Likewise, element $D$ shares one node with element $A$ and is therefore replaced with the template found in Fig. 15(a). Element $E$ does not share any nodes with Element $\mathrm{A}$ and is therefore not replaced with a refinement template. Figure 16 shows the mesh resulting from refining the mesh in Fig. 14 in this manner.

Although Fig. 16 is a valid mesh, wedges and pyramids have been introduced in the transition region. The template in Fig. 15(a) introduces a wedge in the transition region and the template in Fig. 15(b) introduces a wedge and a pyramid in the transition region. In order to maintain tetrahedra in the transition zone these pyramids and wedges must also be converted to tetrahedra. To accomplish this conversion, an additional set of steps must be added to the algorithm in Fig. 5. These steps are shown in Fig. 17.

Step two in Fig. 17 requires looping through the quadrilateral faces of the pyramids and wedges to be converted to tetrahedra. For pyramids, there is only one quadrilateral face. For wedges, there are three

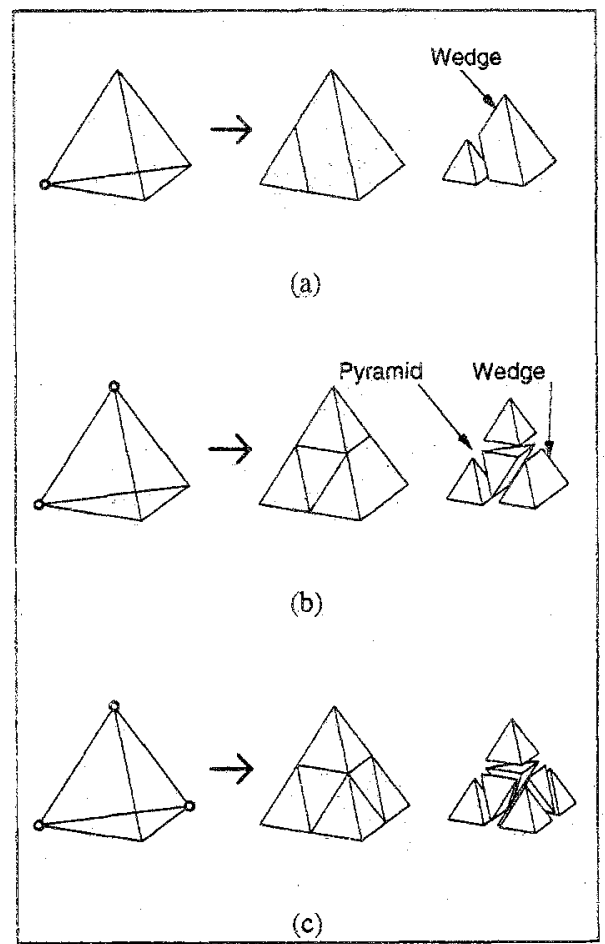

Fig. 15. Tetrahedral retain-element-types transition element templates.

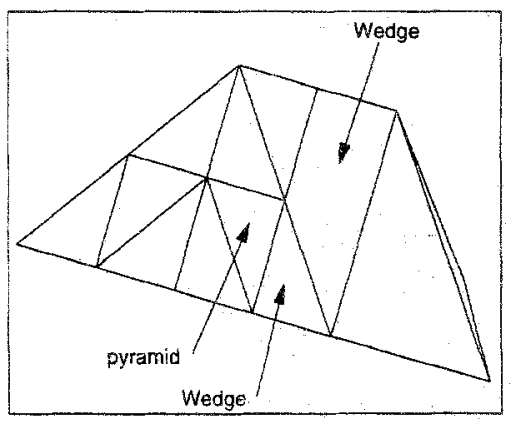

Fig. 16. Figure 14 partially refined using the all-elements-totetrahedra method.

quadrilateral faces. In step two, it must be decided which way to split each quad face. Each quadrilateral face must be split since tetrahedra have no quadrilateral faces. There are many methods for determining which diagonal to split. The Delaunay criterion could be used, although this could lead to problems if the quadrilateral face is not planar. A simpler test is to compare the lengths of the two possible diagonals. Choosing the shortest diagonal will normally lead to the best shaped triangles. Assuming that the original elements in the mesh are convex elements, choosing the shortest diagonal will always result in valid elements. 
Steps to eliminate non-tetrahedral

elements from the transition zone.

1. Create a list of the elements which are to be converted to tetrahedra.

2. For each element in the list, loop through each of the quadrilateral faces on the element. For each quadrilateral face, decide along which diagonal the face should be split.

3. Match the combination of quad face diagonal splits with the possible combinations found in Figure 18. If the diagonals match Figure $18 \mathrm{a}$, the refinement template in Figure 19a is used, if the diagonals match Figure $18 \mathrm{~b}$, the refinement template in Figure $19 \mathrm{~b}$ is used, etc.

4. Replace the element with the template identified in step 3.

Fig. 17. Steps to eliminate non-tetrahedral elements from the transition zone of tetrahedral meshes.

It is possible, however, that the lengths of the two possible diagonals are the same. In this case, an area comparison test can be performed. In an area comparison test, one diagonal is chosen and the areas of the triangles that would result by choosing this diagonal are calculated. The absolute value of the difference between these two areas is recorded. Then, the other diagonal is chosen. Again, the areas of the triangles that would result by choosing this second diagonal are calculated and the difference between the areas is recorded. The diagonal that results in the smallest difference in area is chosen since this generally leads to better shaped tetrahedra in the interior of the element.
If both the lengths and the difference in areas are the same for both diagonals, an arbitrary test needs to be performed to determine which diagonal to choose. Under this case, choosing either diagonal will result in tetrahedra of similar quality. However, a repeatable criterion must be used to choose between the diagonals. This is necessary since each quadrilateral face may be shared by two elements. For example, the template in Fig. 15(b) introduces a pyramid and a wedge that share a common quadrilateral face. Similarly if two adjacent transition tetrahedra are each replaced by the template in Fig. 15(a), two wedges will be introduced that also share a common quadrilateral face. Disjoint faces will result if one diagonal is chosen when analyzing the quadrilateral face coming from one of the elements and the other diagonal is chosen when analyzing the face coming from the other element. The author suggests that the data structure of each node contains a unique node id. The diagonal that contains the node with the highest node id could be chosen. This node id test guarantees that the same diagonal is chosen regardless of from which element the quad face is analyzed.

Once it is decided how each of the quadrilateral faces will be split, step three in Fig. 17 requires that one of the combinations of diagonals in Fig. 18 be chosen. Since only wedges and pyramids are introduced in the transition regions of tetrahedral meshes, Fig. $18(\mathrm{~g}, \mathrm{~h}, \mathrm{i})$ can be ignored for now. After matching the diagonals with one of the cases in Fig. 18, the associated template must be identified. If Fig. 18(a) matches, then the template in Fig. 19(a) is used, if Fig. 18(b) matches, then the template in Fig. 19(b) is used, etc. With the exception of the template defined in Figs 19(c) and 19(d), the wedges and pyramids can be converted to tetrahedra without introducing any new nodes. Step three in Fig. 17 finishes the elimination of the wedges and pyramids from the transition regions by replacing each wedge and pyramid with the template identified in step two. Figure 20 shows the same tetrahedral mesh as in Fig. 14 after refining and eliminating the wedges and pyramids from the transition region.

\section{All-Elements-to-Tetrahedra-Coarse Method of Refinement}

The algorithm described in the previous section for eliminating wedges and pyramids from the transition region of tetrahedral meshes (Fig. 17) can also be used to perform a variation of the all-elements-totetrahedra method of refinement. In the all-elementsto-tetrahedra method of refinement described above, 




Fig. 18. Possible diagonal combinations for conversion of any element type to tetrahedra.

each hexahedra was subdivided into 48 tetrahedra, each wedge was subdivided into 24 tetrahedra, each pyramid was subdivided into 16 tetrahedra, and each tetrahedra was subdivided into eight smaller tetrahedra. This results in a suitable refinement if a high degree of refinement is desired. Often, however, a coarser refinement is preferable. A coarser refinement is possible with the all-elements-to-tetrahedra-coarse method of refinement.

Figure 21 summarizes the steps in the all-elementsto-tetrahedra-coarse method of refinement. Unlike the other refinement algorithms, the all-elements-totetrahedra-coarse method is not usually used to refine only selected portions of a mesh. In step two in Fig. 21 , each non-tetrahedral element is replaced by one of the templates in Fig. 19.

The template defined in Fig. 19(i) is the 'catch all template'. The possible combinations of ways to divide the six faces of a hexahedron are much more numerous than the two presented in Fig. 18. There are, however, only a few combinations of diagonals possible on a hexahedron which can be converted to tetrahedra without introducing a new node at the centroid of the hexahedron. Two of these combinations are shown in Fig. 18(g) and Fig. 18(h). Figure 19(i) is used as the 'catch all template' because it is used to convert any hexahedron that does not match either Fig. $18(\mathrm{~g})$, or Fig. 18(h). The template in Fig. 19(i) is applied by inserting a new node at the center of the hexahedron and creating six pyramids (one from each of the six quadrilateral faces of the hexahedron with the new node at the centroid as the apex of each new pyramid). Each pyramid is then split into two tetrahedra using the template defined in Figs 19(a) or 19(b), depending upon which way each of the diagonals of the respective quadrilateral faces is split.

It could be argued that the all-elements-totetrahedra-coarse method of refinement is actually not a refinement method. For the most part, the accuracy of the solution from a finite element solver is a function of the number of nodes in a mesh rather than the number of elements. Since the all-elements-totetrahedra-coarse method adds only a minimal number of nodes to the mesh, the accuracy of the solution is not generally improved. Many finite solvers, however, require that the mesh be composed strictly of tetrahedra. If this is the case, the all-elements-to-tetrahedra-coarse method can be used to convert any mesh, composed of any of the four standard mesh elements, into a mesh composed of entirely tetrahedra. Therefore, since the main purpose of the all-elements-to-tetrahedra-coarse method is to convert the mesh into tetrahedra, there is rarely the need to use the all-elements-to-tetrahedra-coarse method on only a selected portion of the mesh. Although this method can be extended to convert only a selected portion of the mesh to tetrahedra, the templates to do so are not included here.

\section{Conclusions}

Several general purpose three-dimensional refinement algorithms have been introduced. The algorithms are general enough to refine a finite element mesh composed of any combination of the four standard element types (i.e. hexahedron, wedge, pyramid, or tetrahedron). Either the entire mesh or only a selected portion of the mesh can be refined. The algorithms are simple, easy to implement, and can be implemented either external to, or within a finite element solver. The only difficulty is the large number of transition element refinement templates. However, each of the templates necessary has been defined and outlined by 


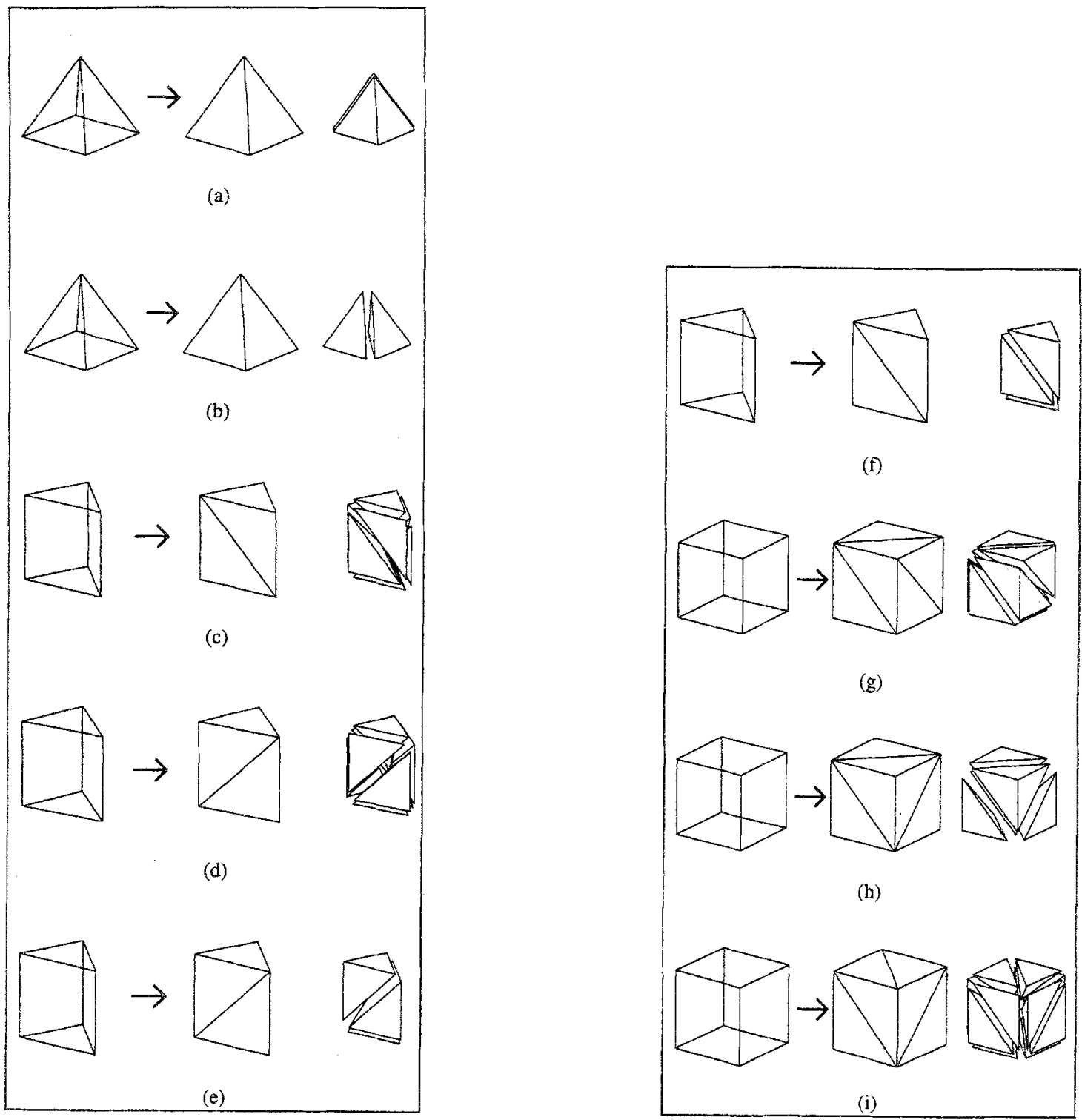

Fig. 19. All-elements-to-tetrahedra-coarse method refinement templates.

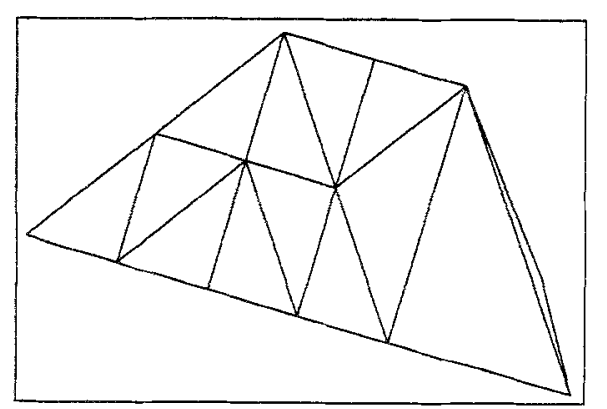

Fig. 20. Figure 14 refined using the all-elements-to-tetrahedra method.

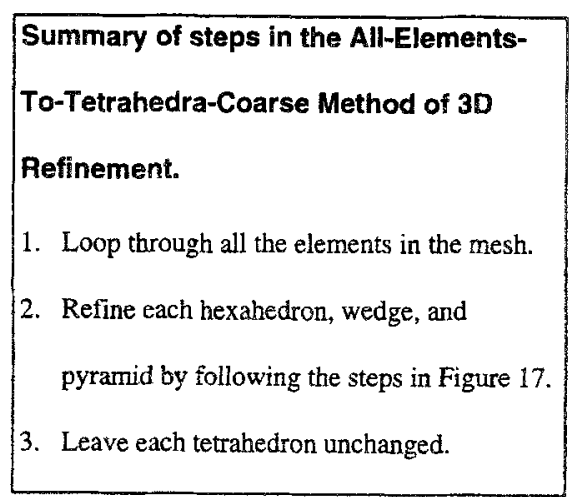

Fig. 21. Summary of steps in all-elements-to-tetrahedra-coarse method of $3 D$ refinement. 
Staten [6]. A copy of each template can be down loaded from the internet at http://www.et.byu.edu/ geos/refine.html.

The algorithms presented in this paper have been implemented in The Department of Defense Groundwater Modeling System (GMS). GMS is a pre- and post-processor to the finite element analysis code FEMWATER. The implementation in GMS is independent of any finite element solver, although the algorithms could be implemented directly in a solver.

\section{References}

1. Ning; Yu-Wei; Suprobo, P.; Jeong, G.D.; Ting, E.C. (1993) A regional mixed refinement procedure for finite element mesh design. Finite Elements in Analysis and Design, 13, 299-318

2. Rivara, M. (1991) Local modifications of meshes for adaptive and/or multigrid finite-element methods. Journal of Computational and Applied Mathematics, 36, 79-89.

3. Kallinderis, Y; Vijayan, P. (1993) Adaptive refinementcoarsening scheme for three-dimensional unstructured meshes. AIAA Journal, 31, 8, 1440-1447

4. Lewis, R.W; Zheng, Y.; Usmani, A.S. (1995) Aspects of adaptive mesh generation based on domain decomposition and Delaunay triangulation. Finite Element Meshes in Analysis and Design, $20,47-70$

5. Schneiders, R.; Debye, J. (1995) Refinement algorithms for unstructured quadrilateral or brick element meshes. Proceedings IMA Workshop on Modeling, Mesh Generation and Adaptive Numerical Methods for Partial Differential Equations

6. Staten, M.L. (1996) Selective refinement of two and three-dimensional finite element meshes, Master's Thesis, Brigham Young University, April 1996, http:/www.et.byu/ geos/refine.html 